\title{
Forschendes Lernen in der curricularen Verankerung in der Medizin
}

\section{Sabine Gronewold, Klaus Peter Kohse und Kirsten Gehlhar}

Forschendes und forschungsorientiertes Lernen (Huber, 2014) findet im Medizinstudium in Deutschland bereits in verschiedenen Modellen statt. Im Hinblick auf die internationale Hochschulausbildung, gemäß Bachelor-Master-Modell mit entsprechenden Forschungsleistungen, ist es aber noch deutlich zu verstärken. Die Approbationsordnung für Ärzte ( ̈̈ApprO) sieht keine in das Studium integrierte Forschungsleistung vor und die Fülle der geforderten Leistungsnachweise erschwert Fakultäten eine Schwerpunktsetzung und systematische Ausbildung im Bereich Wissenschaft und Forschung. Meist bearbeiten Studierende erst im Rahmen ihrer Promotion eigenständig ein wissenschaftliches Projekt und stehen hier teils vor enormen Herausforderungen bei der Durchführung (Sennekamp et al., 2016).

Der Modellstudiengang Humanmedizin wurde zum Wintersemester 2012/13 an der Universität Oldenburg auf Basis des Lehrkonzepts „G2010“ (Kuks 2010) des Bachelor-Master-Studiengangs Medizin der Universität Groningen eingerichtet. Die Studierenden sollen auf Basis des CanMEDS-Rahmenmodells der „Canadian Medical Education Directives for Specialists" (Frank \& Danoff, 2007) in allen im ärztlichen Beruf erforderlichen Kompetenzen trainiert und auf den Beruf vorbereitet werden. Die dort beschriebenen professionellen Rollen der Ärztinnen und Ärzte (u. a. als Forschende) leiten sich aus der Berufswelt und den Anforderungen der Gesellschaft ab. Dieses Rahmenmodell ist auch integraler Bestandteil des deutschen „nationalen kompetenzbasierten Lernzielkatalog Medizin“(NKLM) (MFT, 2015). Die Forschungs- bzw. Wissenschaftskompetenz ist dort als ein Schwerpunkt im 
Studium abgebildet (MFT, 2015, Säule 14a „Medizinisch-wissenschaftliche Fertigkeiten"). Zu diesem zählen eine methodische Grundausbildung in allen Bereichen der Forschung, der Epidemiologie sowie der evidenzbasierten Medizin und auch der Erwerb einer „wissenschaftlichen Haltung “ sowie die Fähigkeit zum selbstständigen wissenschaftlichen Denken und Handeln (siehe MFT, 2015, Kap. 6, 7, 11 und 14a).

Das Oldenburger Curriculum zeichnet sich durch eine integrierte, fächerübergreifende, forschungsorientierte, kompetenz- und patientenbasierte Ausbildung aus. Die ersten drei Studienjahre sind in jeweils vier zehnwöchige Module aufgeteilt (siehe Abb. 1), die grundlagenwissenschaftliche und medizinisch-klinische Inhalte integriert vermitteln und in jedem Modul eine Woche für praktische Erfahrungen (u. a. Forschungspraktika) vorsehen.

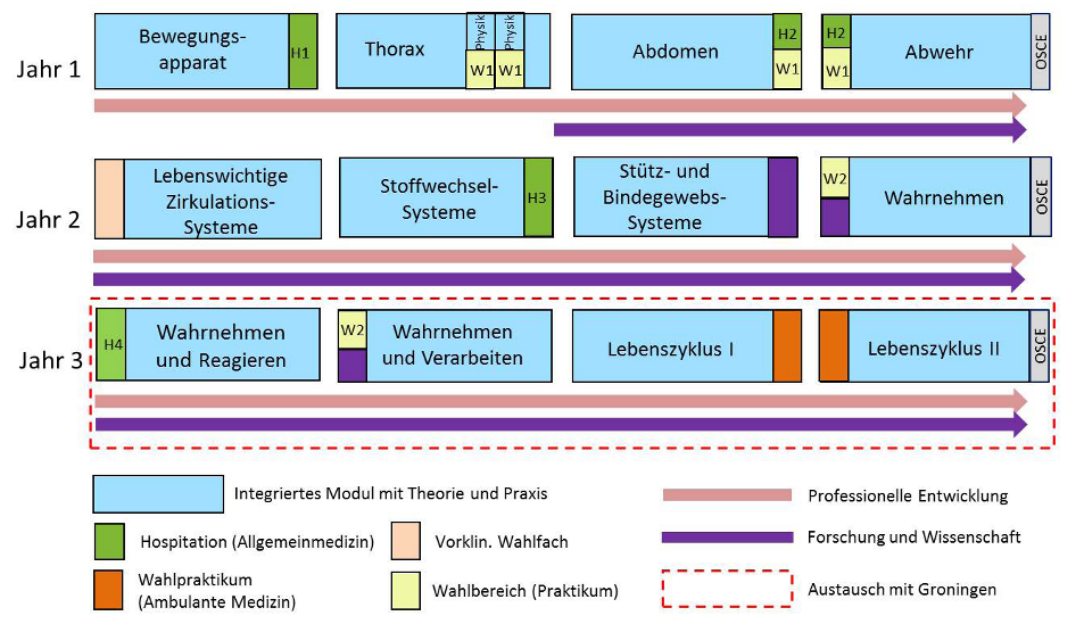

Abb. 1 Studienjahre 1-3 im Modellstudiengang Humanmedizin Oldenburg

Vier inhaltliche Schwerpunkte des Oldenburger Studiengangs werden in kompetenzorientierten, longitudinalen Ausbildungspfaden abgebildet: Kommunikation, klinische Untersuchung, wissenschaftliches Arbeiten (longitudinales Forschungscurriculum $=$ LFC) und professionelle Entwicklung.

Im vierten Studienjahr nimmt der Praxisanteil zu und die Bausteine der ersten drei Jahre (klinisches Wissen, Kommunikation, Untersuchung) werden zusammengeführt. Vier fünfwöchige Blöcke werden in Kliniken absolviert und durch 
fünfwöchige Propädeutika im sogenannten klinischen Trainingszentrum vorbereitet, wo unter anderem die klinisch-praktischen und kommunikativen Fertigkeiten trainiert werden. Im fünften Ausbildungsjahr verbringen die Studierenden fünf vierwöchige Blöcke in klinischen Abteilungen und fertigen eine Forschungsarbeit an. Das sechste Studienjahr bildet das Praktische Jahr (PJ; siehe Abb. 2).

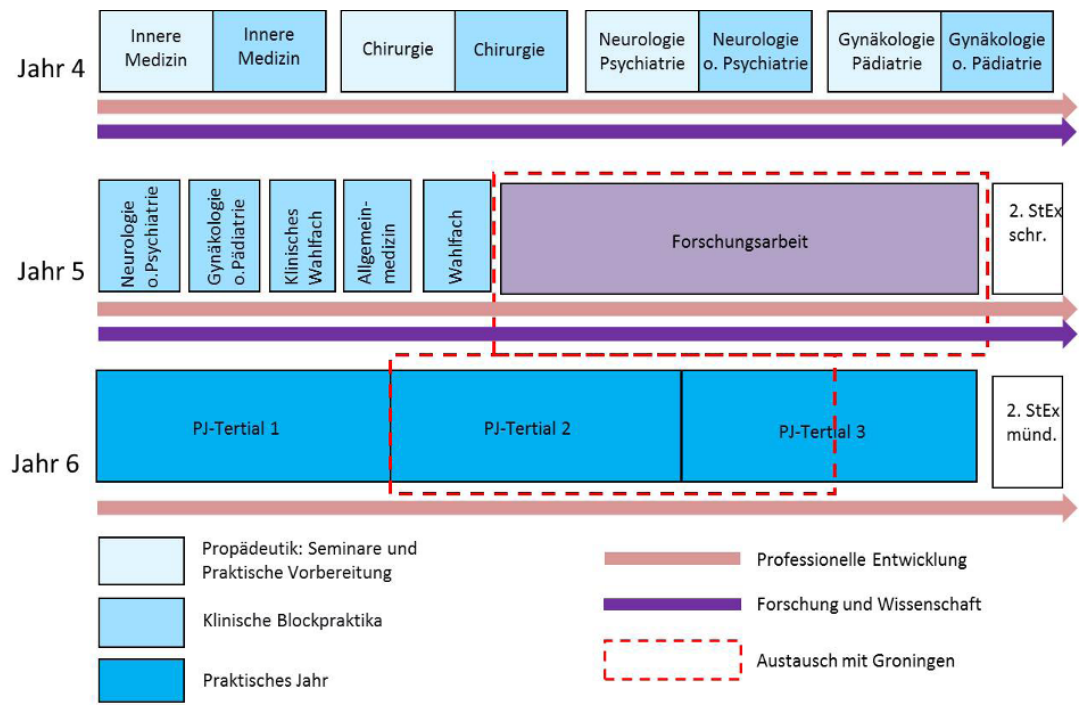

Abb. 2 Studienjahre 4-6 im Modellstudiengang Humanmedizin Oldenburg

Die Einbindung der Studierenden in Wissenschaft und Forschung zieht sich als longitudinales Element („longitudinales Forschungscurriculum“, LFC) durch das gesamte Studium. Ziel ist, die Studierenden in wissenschaftlichem Denken anzuleiten, ihnen Techniken wissenschaftlichen Arbeitens zu vermitteln, Einblicke in aktive Forschungstätigkeit zu geben und sie für die Forschung zu begeistern (siehe Abb. 3). 

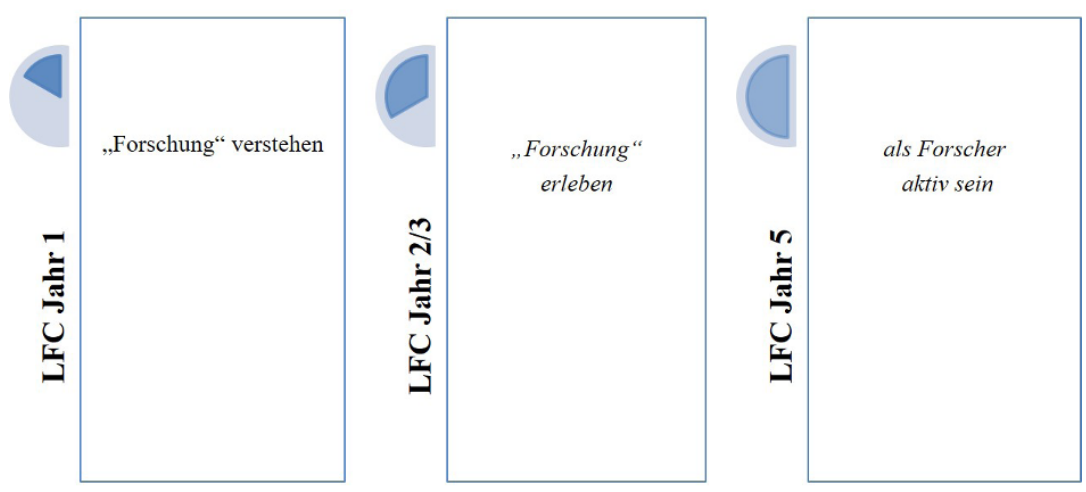

Abb. 3 Die Phasen des longitudinalen Forschungscurriculums (LFC)

\section{LFC Jahr 1}

Im zweiten Semester bearbeiten Studierende in Kleingruppen eine eng umschriebene wissenschaftliche Fragestellung und lernen, diese unter Anwendung von Statistik sowie angemessener Forschungsmethoden zu beantworten und erhaltene Ergebnisse zu präsentieren. Sie folgen der Systematik wissenschaftlicher Publikationen: Planung einer Untersuchung, Untersuchungsmethodik, Datensammlung und -analyse, Interpretation und Relativierung der Befunde. Im Einzelnen werden dafür sieben Kleingruppensitzungen, ein einwöchiges Forschungspraktikum, eine Posterpräsentation sowie ein Begleitseminar mit wichtigen, grundlegenden Themen durchgeführt.

Der Rahmen für die Arbeit in Kleingruppen wird von Mentorinnen und Mentoren gestaltet, die auch die Studierenden bei der Bearbeitung des Forschungsprojektes begleiten. Sie geben das Thema der Gruppenarbeit vor, wobei zum Teil ein bereits erhobener Datensatz verwendet wird. Die Studierenden erarbeiten sich anhand dieser Vorgaben verschiedene theoretische Bereiche. Am Ende des Moduls wird ein Poster mit einem englischsprachigen Abstract auf einem Symposium präsentiert.

\section{Methodische, forschungsorientierte Ausbildung und LFC Jahr 2/3}

In den Studienjahren 2 und 3 erfolgt die weitere methodische Ausbildung. Grundlagen der erlernten Statistik und Forschungsmethodik werden mit einer strukturierten Anwendung auf ein Datenanalyseprojekt verknüpft. Die Studierenden erstellen einen Plan zur Analyse eines vorgegebenen Datensatzes, lernen diesen 
konkret zu beschreiben, wenden einfache Auswertungsmethoden an und erstellen einen Abschlussbericht.

Im Jahr 4 wird die Ausbildung in Journal Clubs fortgesetzt, in denen Studierende wissenschaftliche Texte lesen, Studien und deren Ergebnisse verstehen und interpretieren. Inhalte der Einführungsveranstaltungen werden dabei wiederholt, angewendet und erweitert.

Zudem wird den Studierenden die Möglichkeit gegeben, in zwei einwöchigen Forschungspraktika einen ersten Einblick in unterschiedliche Forschungsbereiche zu erhalten. Forschungsbezogene Aktivitäten wie zum Beispiel der Besuch einer Summer School können dabei angerechnet werden. Die Hospitationen werden von den Studierenden selbstständig ausgewählt. Dabei sollen sie Forschungsaktivitäten und -prozesse der verschiedenen Einrichtungen sowie die dort angewendeten Methoden kennenlernen. Zur Vorbereitung auf sowohl die Forschungspraktika als auch das spätere wissenschaftliche Arbeiten wird in diesem Abschnitt des Studiums eine Seminarreihe angeboten, welche die theoretischen Grundlagen des ersten Studienjahres vertieft und zusätzlich weitere relevante Themen behandelt. Hierzu zählen eine Einführung in die „gute wissenschaftliche Praxis“, rechtliche Aspekte, Versuchsplanung/Studiendesign, experimentelles Arbeiten, Lesen von wissenschaftlichen Texten, wissenschaftliches Schreiben sowie Auswirkungen und Konsequenzen von Wissenschaft und Forschung auf die Medizin.

\section{LFC Jahr 5}

Im fünften Studienjahr bearbeiten die Studierenden in einem Block von 20 Wochen eigenständig ein größeres Forschungsprojekt. Sie können den Themenbereich frei wählen. Bei der Durchführung wird insbesondere Wert gelegt auf die Formulierung einer Forschungsfrage sowie die Aufstellung überprüfbarer Hypothesen, den kritischen Umgang mit Informationsquellen und Abschätzung der Relevanz für die eigene Fragestellung, methodisch einwandfreies Versuchsdesign, verständliche Darstellung und schlüssige Interpretation der Ergebnisse, inhaltlich korrekte Schlussfolgerungen und Einordnung der eigenen Arbeit in den Kontext des relevanten Forschungsbereichs.

Studierende, die im Anschluss an ihr Studium eine Promotion anstreben, können dazu ein auf dem Forschungsprojekt des 5. Jahres aufbauendes Thema bearbeiten. Begleitend werden passende Wahl-Seminare angeboten. 


\section{Forschungsorientierte Lehre im Curriculum des Modellstudiengangs Humanmedizin}

Weitere Elemente des Curriculums stellen ebenfalls Bezüge zu Wissenschaft und Forschung her und sollen forschungsorientiertes Lernen anregen. So werden im problemorientierten Lernen (POL) Fallbeispiele angeboten, die eine Auseinandersetzung mit aktueller Forschungsliteratur erfordern. Die Veranstaltungsreihe „Stand der Wissenschaft" vermittelt einen Überblick über den aktuellen Forschungsstand in verschiedenen Fachbereichen und ermöglicht Einblicke in aktuelle Forschungstätigkeiten an der Universität.

Im Jahr 5 wird mit dem Lehrformat „PICO“ ein klinisches Instrument der evidenzbasierten Entscheidungsfindung eingeführt. Es enthält ein Gerüst aus den vier Bestandteilen P (Patient/Problem; Darstellung des Patienten/des Grundproblems/ der Erkrankung), I (Intervention; Beschreibung der Intervention/Maßnahme), $C$ (Comparison; Beschreibung der Vergleichsmaßnahme(n)) und O (Outcome; Beschreibung der Endpunkte, an denen man Erfolg messen möchte). Diese Schritte sind wesentliche Bausteine der Entscheidungsfindung im klinischen Alltag: eine schnelle und zuverlässige Literatursuche durchführen, die wissenschaftliche Validität und Relevanz der gefundenen Studie/Evidenz bewerten und im Anschluss die Ergebnisse auf den Fall, also den Patienten oder die Patientin, anwenden. Die Studierenden lernen so, dass auch im klinischen Alltag ein forschungsorientiertes Handeln zwingend ist.

\section{Promotionsphase}

Studierende haben in Oldenburg die Möglichkeit, bereits nach dem sechsten Semester ein Promotionsvorhaben anzumelden und studienbegleitend durchzuführen. Die in den Naturwissenschaften vorhandene Infrastruktur (Graduiertenkolleg, Forschungskolloquien etc.) kann dabei von den Promovenden in der Humanmedizin mitgenutzt werden; sie wird derzeit deren Bedürfnissen durch eine Erweiterung der Veranstaltungsangebote angepasst. Gemäß den Empfehlungen des Wissenschaftsrates (Wissenschaftsrat, 2018) wird ein strukturiertes Promotionsprogramm etabliert, das inhaltlich an das longitudinale Forschungscurriculum anknüpfen und den „roten Wissenschafts-Faden“ bis in die Phase der wissenschaftlichen Weiterqualifikation fortführen wird. 


\section{Kritische Diskussion und Fazit}

Der Wissenschaftsrat hat sich bereits im Jahr 2014 deutlich für eine Stärkung der wissenschaftlichen Kompetenzen im Medizinstudium ausgesprochen, betont deren Bedeutung für eine verantwortungsvolle ärztliche Berufsausübung und empfiehlt ihren Erwerb als einen zentralen, idealerweise aufbauend konzipierten Baustein der studentischen Ausbildung (Wissenschaftsrat, 2014).

Die Empfehlungen zum Masterplan Medizinstudium 2020 (Wissenschaftsrat, 2018) enthalten ebenfalls einen longitudinalen Pfad zum Erwerb wissenschaftlicher Kompetenzen im Kerncurriculum, die in einem Wahlcurriculum vertieft werden. Zudem werden ein verpflichtender wissenschaftlicher Leistungsnachweis vor dem ersten Abschnitt der Ärztlichen Prüfung und ein umfangreicherer zweiter vor dem zweiten Abschnitt empfohlen. Dabei sollen die einzelnen Bausteine eines Forschungscurriculums sichtbar und untereinander abgestimmt sein sowie Querbezüge zu verwandten curricularen Veranstaltungen aufweisen.

Der Oldenburger Modellstudiengang bildet diese wesentlichen Bausteine der wissenschaftlichen Ausbildung bereits longitudinal ab. Allerdings betrachten Studierende die verschiedenen Elemente oft als isolierte Bausteine ohne Zusammenhang. Erste Evaluationsergebnisse zum LFC Jahr 1 zeigen, dass der Transfer von der Theorie zur Praxis schwerfällt. Der frühe Einblick in die wissenschaftliche Praxis begeistert, aber die notwendigen theoretischen Hintergründe sind nur schwer einheitlich zu vermitteln. Hier werden motivierte und für das Konzept geschulte Lehrende benötigt.

Die Veranstaltungsangebote und geforderten Leistungen zum Erwerb wissenschaftlicher Kompetenzen scheinen auch im weiteren Verlauf des Studiums als „Insel-Wissen“ wahrgenommen zu werden. Studierende melden einen Mangel an Wissen und Kompetenzen, die sie sich problembezogen selbst aneignen müssen. Diese Wahrnehmung geht mit einer subjektiv sehr hoch empfundenen Arbeitsbelastung einher. Inwieweit in den späteren Studienabschnitten auf das bereits erworbene Wissen tatsächlich zugegriffen und dieses angewendet werden kann, lässt sich derzeit noch nicht bestimmen.

Um hier eine Verbesserung zu erreichen, wurden die einzelnen Bausteine evaluiert, wenn notwendig inhaltlich und vom Umfang her angepasst und inhaltlich besser aufeinander abgestimmt, sodass die erworbenen Fähig- und Fertigkeiten sinnvoll aufeinander aufbauen und ineinandergreifen. Ein "roter Forschungsfaden“, abgebildet in einem selbstständig erstellten Portfolio als „Forschungstagebuch“, soll entstehen, in dem bereits erworbenes Wissen und Kompetenzen zusammengetragen und transparent dargestellt werden. Dieses kann zur Vorbereitung auf die Forschungsarbeit im Jahr fünf und für eine Übersicht über bisher erworbenes 
Wissen und Kompetenzen sowie die eigene Interessen- und Schwerpunktsetzung genutzt werden. Da durch die aktuell gütige Approbationsordnung eine Forschungsleistung nicht abgebildet ist, wird der hierfür zu leistende Aufwand von einigen Studierenden (und z. T. auch Lehrenden) kaum akzeptiert. Im Licht einer Tradition, in der ein humanmedizinisches Promotionsvorhaben bereits während des Studiums begonnen werden kann, wird die im Oldenburger Curriculum vorgesehene Forschungsarbeit im Jahr fünf gelegentlich fälschlich als Promotionsäquivalent betrachtet. Der Prozess der Einführung forschenden und forschungsorientierten Lernens im Humanmedizin-Studium benötigt daher sowohl strukturelle Unterstützung als auch eine intensive fakultäre Schulung und Begleitung, um eine studentische Forschungskultur sowie die dafür notwendigen Rahmenbedingungen und Infrastruktur zu schaffen.

\section{Literatur}

Approbationsordnung für Ärzte (ÄAppO) (2002). Veröffentlicht 27. Juni 2002 (BGBl. I S. 2405), zuletzt geändert durch Art. 5 G v. 17.7.2017, I 2581. Verfügbar unter https://www. gesetze-im-internet.de/_appro_2002/BJNR240500002.html [29.01.2019].

Frank, J. R., Danoff, D. (2007). The CanMEDS 2005 Physician CompetencyFramework: Better standards. Better physicians. Better care. Medical Teacher, 29(7), S. 642-647.

Huber, L. (2014). Forschungsbasiertes, Forschungsorientiertes, Forschendes Lernen: Alles dasselbe? Ein Plädoyer für eine Verständigung über Begriffe und Unterscheidungen im Feld forschungsnahen Lehrens und Lernens. Das Hochschulwesen, 62(1 \& 2) S. 22-29.

Kuks, J. B. M. (2010). The bachelor-master structure (two-cycle curriculum) according to the Bologna agreement: a Dutch experience. Medisch Onderwijs, 29(1), S. 16-21.

Medizinischer Fakultätentag (MFT) (Hrsg.) (2015). Nationaler kompetenzbasierter Lernzielkatalog Medizin (NKLM), Berlin: MFT.

Sennekamp, M., Paulitsch, M. A., Broermann, M., Klingebiel, T., Gerlach, F. M. (2016). Auf dem Weg zum Dr. med.: Welche Unterstützung brauchen Promovierende der Medizin? Teill: Bestandsaufnahme und Konzeptentwicklung. Zeitschrift für Evidenz, Fortbildung und Qualität im Gesundheitswesen,110-111, S.69-76.

Wissenschaftsrat (WR) (2014). Empfehlungen zur Weiterentwicklung des Medizinstudiums in Deutschland auf Grundlage einer Bestandsaufnahme der humanmedizinischen Modellstudiengänge (Drs. 4017-14). Verfügbar unter http://www.wissenschaftsrat.de/download/ archiv/4017-14.pdf [29.01.2019].

Wissenschaftsrat (WR) (2018). Neustrukturierung des Medizinstudiums und Änderung der Approbationsordnung für Ärzte: Empfehlungen der Expertenkommission zum Masterplan 2020 (Drs. 7271-18) Verfügbar unter https://www.wissenschaftsrat.de/download/ archiv/7271-18.pdf [29.01.2019]. 


\section{Zu den Autorinnen und zum Autor}

Sabine Gronewold, Dipl. Psych, ist Referentin für Curriculumsentwicklung im Studiendekanat der Fakultät für Medizin und Gesundheitswissenschaften mit dem Schwerpunkt der wissenschaftlichen Qualifikation der Studierenden im Studium und der Weiterqualifikationsphase.E-Mail: sabine.gronewold@uni-oldenburg.de Prof. Dr. Dr. Klaus Peter Kohse ist Direktor des Instituts für Laboratoriumsdiagnostik und Mikrobiologie am Klinikum Oldenburg und Studiendekan der Fakultät VI Medizin und Gesundheitswissenschaften an der Carl von Ossietzky Universität Oldenburg. E-Mail: klaus.peter.kohse@uol.de

Dr. Kirsten Gehlhar hat einen Masterabschluss im Bereich Medical Education und ist Leiterin des Studiendekanats und stellvertretende Studiendekanin der Fakultät für Medizin und Gesundheitswissenschaften an der Carl von Ossietzky Universität Oldenburg. Ihre Arbeitsschwerpunkte liegen in den Bereichen der curricularen Weiterentwicklung. E-Mail: kirsten.gehlhar@uni-oldenburg.de

Open Access Dieses Kapitel wird unter der Creative Commons Namensnennung 4.0 International Lizenz (http://creativecommons.org/licenses/by/4.0/deed.de) veröffentlicht, welche die Nutzung, Vervielfältigung, Bearbeitung, Verbreitung und Wiedergabe in jeglichem Medium und Format erlaubt, sofern Sie den/die ursprünglichen Autor(en) und die Quelle ordnungsgemäß nennen, einen Link zur Creative Commons Lizenz beifügen und angeben, ob Änderungen vorgenommen wurden.

Die in diesem Kapitel enthaltenen Bilder und sonstiges Drittmaterial unterliegen ebenfalls der genannten Creative Commons Lizenz, sofern sich aus der Abbildungslegende nichts anderes ergibt. Sofern das betreffende Material nicht unter der genannten Creative Commons Lizenz steht und die betreffende Handlung nicht nach gesetzlichen Vorschriften erlaubt ist, ist für die oben aufgeführten Weiterverwendungen des Materials die Einwilligung des jeweiligen Rechteinhabers einzuholen.

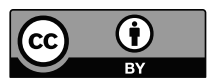

